\title{
Density functional theory of superconductivity in doped tungsten oxides
}

\author{
Camilla Pellegrini, ${ }^{1, *}$ Henning Glawe, ${ }^{2}$ and Antonio Sanna ${ }^{1, \dagger}$ \\ ${ }^{1}$ Max Planck Institute of Microstructure Physics, Weinberg 2, 06120 Halle, Germany \\ ${ }^{2}$ Max Planck Institute for the Structure and Dynamics of Matter, Luruper Chaussee 149, 22761 Hamburg
}

(Received 22 March 2019; published 24 June 2019)

\begin{abstract}
We apply density functional theory for superconductors (SCDFT) to doped tungsten oxide in three forms: electrostatically doped $\mathrm{WO}_{3}$, perovskite $\mathrm{WO}_{3-x} \mathrm{~F}_{x}$, and hexagonal $\mathrm{Cs}_{x} \mathrm{WO}_{3}$. We achieve a consistent picture in which the experimental superconducting transition temperature $T_{c}$ is reproduced, and superconductivity is understood as a weak-coupling state sustained by soft vibrational modes of the $\mathrm{WO}_{6}$ octahedra. SCDFT simulations of $\mathrm{Cs}_{x} \mathrm{WO}_{3}$ allow us to explain the anomalous $T_{c}$ behavior observed in most tungsten bronzes, where $T_{c}$ decreases with increasing carrier density. Here, the opening of structural channels to host Cs atoms induces a softening of strongly coupled W-O modes. By increasing the Cs content, these modes are screened and $T_{c}$ is strongly reduced.
\end{abstract}

DOI: 10.1103/PhysRevMaterials.3.064804

\section{INTRODUCTION}

Tungsten trioxide $\mathrm{WO}_{3}$, its bronzes $\mathrm{M}_{x} \mathrm{WO}_{3}$, and related compounds, e.g., oxygen-deficient $\mathrm{WO}_{3-x}$ and fluorine-doped $\mathrm{WO}_{3-x} \mathrm{~F}_{x}$, are a family of crystals of high scientific interest and potential technological impact. The main focus is on their electrochromic properties for smart windows applications [1], but a number of other significant properties are known [2,3], among which is superconductivity.

Superconductivity of tungsten bronzes was first discovered by Raub et al. [4] for $M=\mathrm{Na}$ at $x \simeq 0.3$ with a critical temperature $T_{c}=0.55 \mathrm{~K}$. This observation has been confirmed by similar measurements for various alkali dopings [5-7], also in recent accurate studies [8], and extended to indium-based compounds [9], for which $2 \mathrm{~K}<T_{c}<4 \mathrm{~K}$ and to $\mathrm{WO}_{3-x} \mathrm{~F}_{x}$ [10] with $T_{c} \simeq 0.5 \mathrm{~K}$.

To this consistent scenario of low $-T_{c}$ superconductivity, some reports have been added on possible low-dimensional and metastable high- $T_{c}$ superconductivity upon surface doping of $\mathrm{WO}_{3}$ crystals with sodium [11] and hydrogen [12], as well as at the $\mathrm{W} / \mathrm{WO}_{3}$ interface [13].

Since their discovery, the unusual features of the superconducting state in tungsten bronzes have given rise to a large number of investigations and tentative interpretations. As a matter of fact, several questions are still waiting for a consistent answer: (i) Are the stable low-temperature superconducting phases driven by conventional electron-phonon coupling? Or, like in $\mathrm{BaBiO}_{3}$, conventional approaches fail, and the correct pairing mechanism is still an open problem? (ii) What is the physical origin of the $T_{c}$ "paradox" observed in tungsten bronzes, i.e., the decrease of $T_{c}$ with increasing doping and density of states (DOS) at the Fermi level? (iii) Can conventional electron-phonon coupling, in some geometries

\footnotetext{
*camilla.pellegrini@mpi-halle.mpg.de

†sanna@mpi-halle.mpg.de
}

and doping regimes, provide enough coupling strength to lead to high $T_{c}$ ?

Even though a few theoretical investigations have attempted to provide a comprehensive picture, research has mostly focused on structural and electronic properties, without specifically addressing the superconducting state. However, a systematic experimental study has recently raised the need for a comprehensive theoretical analysis of superconductivity in this family of compounds [8].

In this work we are filling the gap by providing a full $a b$ initio characterization of doped $\mathrm{WO}_{3}$ by state-of-theart (Sec. II) density functional theory for superconductivity (SCDFT). To this end, we start (Sec. III A) by considering the low-temperature structural phase of $\mathrm{WO}_{3}$, to which we apply electrostatic doping, in order to probe the intrinsic coupling of the $\mathrm{WO}_{3}$ tetrahedron. Then (Sec. III B) we investigate the effect of chemical doping, i.e., the joint effect of electrostatic doping, lattice distortions, and extrinsic contributions from impurity lattice modes. The two forms of chemical doping that we have chosen are fluorine substitutions (Sec. III B 1), that retain the perovskite geometry, and Cs intercalations, as representative of the large family of hexagonal tungsten bronzes (Sec. III B 2).

\section{METHODS AND COMPUTATIONAL DETAILS}

The electronic properties of bulk $\mathrm{WO}_{3}$ in different lattices have been investigated extensively in the literature. Two of the most complete works on the subject are the early paper by de Wijs and co-workers [14], which employs a density functional theory-generalized gradient approximation (DFT-GGA) approach, and a recent paper by Hamdi and co-workers [15], based on the B1-WC approximation, i.e., a hybrid-functional approach, specifically tuned to describe perovskite oxides. Even though the B1-WC method delivers lattice parameters that are overall more accurate than GGA, these two approaches give qualitatively similar results and, in particular, agree on the low-temperature lattice structure. As 
for the present work, in order to be able to compute lattice dynamical properties at an affordable computational cost, we have adopted the DFT-GGA approximation.

Computational details. All electron and phonon calculations have been performed with the Quantum ESPRESSO [16] package in the norm-conserving pseudopotential approximation, with a plane-wave expansion cutoff of $80 \mathrm{Ry}$. $\mathbf{k}$, q-point sampling has been chosen for each compound on the basis of convergence tests (see below). Superconducting properties have been computed using SCDFT [17-20], as recently reviewed in Refs. [21] and [22].

In SCDFT, both the electronic density $n(\mathbf{r})$ and the order parameter $\chi\left(\mathbf{r}, \mathbf{r}^{\prime}\right)$ of the system are reproduced by a superconducting noninteracting Kohn-Sham (KS) system, where the exchange-correlation effects on superconductivity are contained in the effective pairing potential $\Delta_{s}\left(\mathbf{r}, \mathbf{r}^{\prime}\right)$, which is a functional of $n(\mathbf{r})$ and $\chi\left(\mathbf{r}, \mathbf{r}^{\prime}\right)$. Within the decoupling approximation [18,23] (that is exact in the vicinity of $T_{c}$ ), the calculation of the electron density and phonon properties is effectively decoupled from the calculation of the superconducting properties of the system. As a consequence, the former can be computed in the normal state (NS) with conventional codes [16,24], while the latter are obtained by solving self-consistently a BCS-like equation, i.e., the SCDFT gap equation [18]:

$$
\Delta_{s}^{n \mathbf{k}}=\mathcal{Z}_{n \mathbf{k}} \Delta_{s}^{n \mathbf{k}}-\sum_{n^{\prime} \mathbf{k}^{\prime}} \mathcal{K}_{n^{\prime} \mathbf{k}^{\prime}}^{n \mathbf{k}} \frac{\tanh \left[\frac{\beta E_{n^{\prime} \mathbf{k}^{\prime}}}{2}\right]}{2 E_{n^{\prime} \mathbf{k}^{\prime}}} \Delta_{s}^{n^{\prime} \mathbf{k}^{\prime}} .
$$

Here, $\Delta_{s}^{n \mathbf{k}}=\left\langle\varphi_{n \mathbf{k}}\left|\Delta_{s}\right| \varphi_{n \mathbf{k}}\right\rangle$ are the diagonal matrix elements with respect to the NS-KS Bloch orbitals (with band index $n$ and Bloch index $\mathbf{k}), E_{n \mathbf{k}}=\sqrt{\xi_{n \mathbf{k}}^{2}+\left|\Delta_{s}^{n \mathbf{k}}\right|^{2}}$ are BCS quasiparticle energies, where $\xi_{n \mathbf{k}}$ are eigenvalues of the NS-KS system, and $\beta$ is the inverse temperature. The effects of the Coulomb interaction and the retarded electron-phonon coupling are included in the kernels $\mathcal{Z}$ and $\mathcal{K}$, which are implicit functionals of the densities. In practice, these kernels are functionals of the normal electronic and phononic band structure, as well as of the electron-phonon matrix elements and the screened Coulomb interaction.

By assuming that the $n \mathbf{k}$ anisotropy in Eq. (1) is negligible, one can perform iso-energy $(\xi)$ averages of the kernels $\mathcal{Z}$ and $\mathcal{K}$ (see Ref. [18] for further details), which leads to the simplified (isotropic) gap equation:

$$
\Delta_{s}(\xi)=\mathcal{Z}(\xi) \Delta_{s}(\xi)-\int d \xi^{\prime} \mathcal{K}\left(\xi, \xi^{\prime}\right) \frac{\tanh \left[\frac{\beta E\left(\xi^{\prime}\right)}{2}\right]}{2 E\left(\xi^{\prime}\right)} \Delta_{s}\left(\xi^{\prime}\right) .
$$

For all the systems under study we have verified that the above isotropic equation reproduces values of $T_{c}$ which agree with the fully anisotropic ones [obtained from Eq. (1)], within an error of $0.1 \mathrm{~K}$. For this reason, anisotropy effects have been discarded throughout this work.

Within the isotropic approach, the screened Coulomb interaction is accounted for by a function $\mu\left(\xi, \xi^{\prime}\right)$, which is given by the average Coulomb matrix element on the isoenergy surfaces $\xi$ and $\xi^{\prime}$ times the DOS at $\xi^{\prime}$ (see Ref. [25]). At $\xi=$ $\xi^{\prime}=0$ this function takes the value $\mu$ of the Morel-Anderson theory [26,27]. In this work, $\mu\left(\xi, \xi^{\prime}\right)$ has been computed in the static random phase approximation (RPA) within the ELK code [24].

Moreover, the electron-phonon coupling is described in terms of the Eliashberg spectral function [28], defined as

$$
\alpha^{2} F(\omega)=\frac{1}{N_{E_{F}}} \sum_{\mathbf{k q}, v}\left|g_{\mathbf{k}, \mathbf{k}+\mathbf{q}, v}\right|^{2} \delta\left(\epsilon_{\mathbf{k}}\right) \delta\left(\epsilon_{\mathbf{k}+\mathbf{q}}\right) \delta\left(\omega-\omega_{\mathbf{q}, v}\right)
$$

where $N_{E_{F}}$ is the DOS at the Fermi level, $\omega_{\mathbf{q}, v}$ is the phonon frequency of mode $v$ at wave vector $\mathbf{q}$, and $\left|g_{\mathbf{k}, \mathbf{k}+\mathbf{q}, v}\right|$ is the electron-phonon matrix element between two electronic states with momenta $\mathbf{k}$ and $\mathbf{k}+\mathbf{q}$.

At a crude level, as compared to Eq. (1), the superconducting transition temperature can be estimated from McMillan's formula [28-30]:

$$
T_{c}=\frac{\omega_{\log }}{1.2} \exp \left[\frac{-1.04(1+\lambda)}{\lambda-\mu^{*}(1+0.62 \lambda)}\right] .
$$

Here, $\omega_{\log }$ corresponds to a weighted average phonon frequency,

$$
\lambda=2 \int \frac{\alpha^{2} F(\omega)}{\omega} d \omega
$$

is the electron-phonon interaction parameter, and $\mu^{*}$ represents the screened Coulomb interaction between the electrons (of order 0.1). McMillan's formula, thanks to its simple analytic form, will be used in the following as a tool to help us understanding values and trends of the critical temperatures obtained within SCDFT.

\section{RESULTS}

\section{A. Intrinsic coupling: Electrostatically doped $\mathrm{WO}_{3}$}

By using a liquid electrolyte as a gate dielectric, high charge-carrier doping can be experimentally induced in insulating crystals by an electrostatic field effect, triggering an insulator-to-metal transition and superconductivity [31]. Unless complex structural rearrangements are at play [32], electrostatic doping is the cleanest way to accumulate chargecarrier densities on a substrate and probe the intrinsic coupling of a system.

In order to simulate a doping by electrostatic field in $\mathrm{WO}_{3}$, we have computationally added/removed electronic charge from the (bulk) system and introduced a jellium background so as to ensure the charge neutrality of the infinite crystal. Alternatively, one could simulate charge doping by a simple non-self-consistent shift of the Fermi level. However, this latter approach would not account for lattice relaxation effects, which are crucial for the correct description of observed structural transitions [33].

Our results [34] show that the charge doping induces significant changes in the electronic DOS [Fig. 1(a)] and large lattice relaxation effects. While in the undoped $\mathrm{WO}_{3}$ oxygen octahedra are tilted (with an angle of $166 \mathrm{deg}$ between the apical oxygens) but not twisted, hole doping enhances the tilting and induces a twisting, whereas electron doping (already at 0.25 el/f.u.) completely removes the tilting and any monoclinic distortion.

The phonon spectrum of the $\mathrm{WO}_{3}$ system for different doping levels is shown in Fig. 1(b), where it exhibits two 

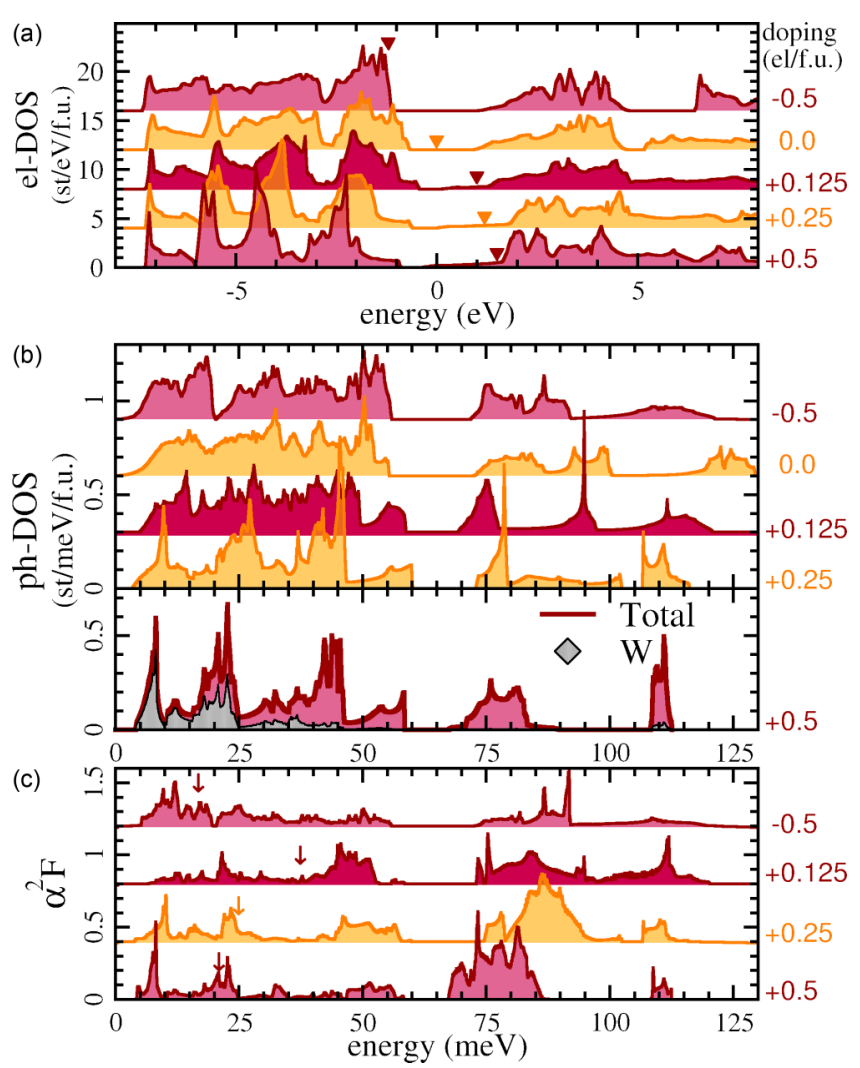

FIG. 1. Collected results for bulk $\mathrm{WO}_{3}$ upon electrostatic doping (jellium approximation), expressed in electrons per formula unit (el/f.u.); vertical shifts are applied for clarity. (a) Electronic density of states (DOS); triangles indicate the Fermi level. (b) Phonon DOS. For the doping level $+0.5 \mathrm{el} /$ f.u. a shaded area represents the projection onto $\mathrm{W}$ vibrational modes. (c) Eliashberg spectral functions; arrows indicate the value of $\omega_{\log }$.

broad structures: a low-energy structure (from 0 to $58 \mathrm{meV}$ at -0.5 el/f.u. doping) and a high-energy structure (from 73 to $110 \mathrm{meV}$ ). At the low end of the low-energy structure, apart from acoustic vibrations, one can identify (at $q=\Gamma$ ) $z$ modes of the tungsten moving in phase with the apical oxygen $\mathrm{O}_{a p}(\sim 19 \mathrm{meV})$, twisting and rocking modes of the octahedron $(\sim 25 \mathrm{meV})$, rocking modes of the $\mathrm{W}-\mathrm{O}_{a p}$ unit $(\sim 28 \mathrm{meV}), x y$ oscillations of the $\mathrm{O}_{a p}(\sim 41 \mathrm{meV})$, and, more in general, modes that do not stretch the W-O bond. Stretching modes of the basal oxygens, $\mathrm{O}_{b a}$, are located at the high end $(60 \mathrm{meV})$ of the low-energy structure and at the low end of the high-energy structure $(78 \mathrm{meV})$. The latter contains all $\mathrm{W}-\mathrm{O}$ stretching modes, namely, those of $\mathrm{W}-\mathrm{O}_{b a}$ in the lowest part and those of $\mathrm{W}-\mathrm{O}_{a p}$ in the highest part of the spectrum. This ends $(113 \mathrm{meV})$ with a full breathing mode of the oxygen octahedron.

Despite this rich lattice dynamics, the superconducting properties of the system are mostly determined by the low end of the phonon spectrum. Specifically, our results show that for doping levels higher than 0.125 , about $60 \%$ of the electron-phonon coupling is provided by the lowest quarter of the spectrum. This follows from the fact that the electronphonon spectral function $\alpha^{2} F(\omega)$ is rather small in the entire frequency range. Then, by taking the weak-coupling limit of
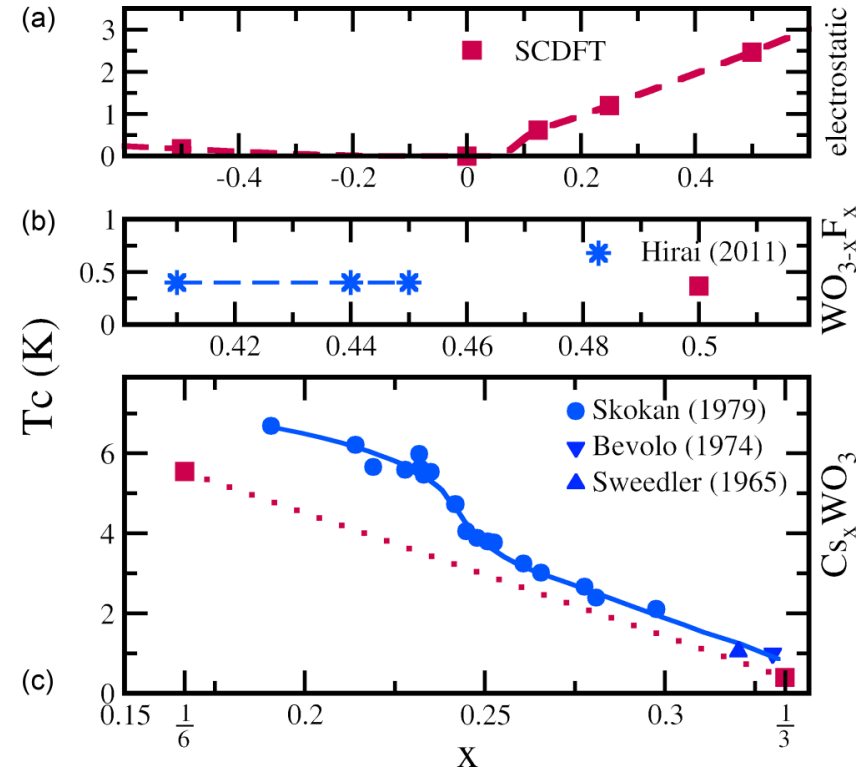

FIG. 2. Superconducting transition temperatures of doped $\mathrm{WO}_{3}$ from SCDFT calculations compared to experimental values. The doping content $x$ is expressed in electrons per $\mathrm{WO}_{3}$ formula unit. (a) electrostatic doping; (b) $\mathrm{F}$ substitution on the $\mathrm{O}$ site; (c) $\mathrm{Cs}$ intercalations.

McMillan's formula in Eq. (4), $T_{c} \sim \exp [-1 /(\lambda-\mu *)]$, one can see that the transition temperature depends exponentially on the average electron-phonon coupling $\lambda$ of Eq. (5), which is weighted towards low frequencies. An indicator of the relevance of the low-energy part of the spectrum is also given by the low value of $\omega_{\text {log }}$, that is marked by an arrow in Fig. 1(c) for the different doping regimes. We also observe that achieving numerical accuracy in simulating this softmode weak-coupling type of superconductivity is particularly difficult since $T_{c}$ is very sensitive to the difference between two small quantities (with associated errors), i.e., the electronphonon $\left(\alpha^{2} \mathrm{~F}\right)$ and the Coulomb interaction $\mu$.

The computed transition temperatures are shown in Fig. 2(a). $T_{c}$ is negligible in the entire hole-doping regime, whereas it increases monotonically with electron doping up to the value of $2.5 \mathrm{~K}$ at $0.5 \mathrm{el} / \mathrm{f}$.u.

This trend is opposite to that experimentally observed in tungsten bronzes [8]. However, since the electron-phonon coupling in the $\mathrm{WO}_{3}$ system is weak, we expect the behavior of $T_{c}$ to be affected not only by variations of the Fermi DOS, which are directly controlled by charge doping, but also by details of the phonon spectrum (at low frequencies). Thus, the results here obtained cannot be straightforwardly generalized to other forms of doping, which also involve size and chemical or disorder effects, since small modifications in the material properties might alter significantly the predicted behavior of $T_{c}$ in Fig. 2(a).

A global treatment of superconductivity in the doped $\mathrm{WO}_{3}$ family clearly requires the accurate description of chemical doping and its effects on the lattice dynamics. We will present this study in the next sections. 


\section{B. Electron-phonon coupling and superconductivity of chemically doped $\mathrm{WO}_{3}$}

Chemical doping of $\mathrm{WO}_{3}$ is effectively achieved by ion intercalation ( $\mathrm{K}[5,6,8], \mathrm{Na}[4]$, In [9], $\mathrm{Cs}[35,36] \ldots)$ and/or substitution [10] $(\mathrm{O} \rightarrow \mathrm{F})$. Introducing large ions leads to significant modifications of the $\mathrm{WO}_{3}$ perovskite structure in which the chains of $\mathrm{WO}_{6}$ octahedra open up to create sufficient interstitial space. Fluorine substitution on the $\mathrm{O}$ sites, on the other hand, preserves the perovskite lattice. Other doping routes are also possible, e.g., $\mathrm{H}$ absorption [37] has been suggested to occur in field experiments at the $\mathrm{WO}_{3}$ surface [38]. All these procedures result in electron doping. Due to the high work function required to extract electrons from the oxygen octahedra [39], hole doping seems unlikely to occur.

\section{1. $\mathrm{WO}_{3-x} \mathrm{~F}_{x}$ at $x=0.5$}

We first consider the case of fluorine substitutional doping. As mentioned in Sec. III A, electron doping tends to stabilize the tetragonal lattice. We then adopt a minimal unit cell with two formula units, which allows us to simulate a doping at fluorine content $x=0.5$. This is close to the maximum experimental value of $x=0.45$ in Ref. [10]. There are two crystallographic sites that fluorine can occupy: apical and planar. The latter is significantly more stable (over $600 \mathrm{meV}$ ) and leads to a stable lattice dynamics. On the contrary, we could not find any dynamically stable structure for fluorine in the apical position [40].

$\mathrm{O} \rightarrow \mathrm{F}$ substitution leads to an effective electron doping of the $\mathrm{WO}_{3}$ system. An atomic orbital projection of the electronic DOS [Fig. 3(a)] shows that the KS states at the Fermi level are about $2 / 3$ on the tungsten site and $1 / 3$ on the oxygen site (with a minor contribution from fluorine). We have reported in Fig. 3(b) the diagonal of the screened Coulomb interaction function, $\mu(\xi, \xi)$. This displays a smooth behavior that follows overall the variations of the DOS. The absence of a peak at the bottom of the doped conduction band points to a good metallic screening, which implies weak Coulomb repulsion at the Fermi level.

As for the case of electrostatic doping (Sec. III A), the phonon spectrum in Fig. 3(c) shows two broad structures. These range from 0 to $55 \mathrm{meV}$ and from 78 to $120 \mathrm{meV}$, respectively, and are separated by a fluorine Einstein mode at $60 \mathrm{meV}$. The low-energy structure $(0-60 \mathrm{meV})$ corresponds to the overlap of several types of lattice vibrations. Below $30 \mathrm{meV}$ most of the spectrum is given by tungsten modes inside the fluorine-oxygen octahedron and by the angular vibrations of the octahedron around the $z$ axis. Between 33 and $40 \mathrm{meV}$ a large contribution to the spectrum comes from fluorine due to oscillations in the plane perpendicular to the $\mathrm{W}-\mathrm{F}$ bond. Between 45 and $57 \mathrm{meV}$ are oxygen modes, where the $\mathrm{W}-\mathrm{O}$ bond is rotating. In addition, the F-W stretching mode gives rise to the Einstein mode at $60 \mathrm{meV}$. W-O stretching modes are all collected in the high-energy part of the spectrum (above $70 \mathrm{meV}$ ), with $\mathrm{W}-\mathrm{O}_{a p}$ stretching at the bottom (72-79 meV) and $\mathrm{W}-\mathrm{O}_{b a}$ stretching vibrations at the top. Since this trend is opposite to the one computed in the absence of fluorine, it indicates that fluorine substitution significantly softens the vibrations of the apical oxygens. This leads to the key feature for superconductivity, that is,

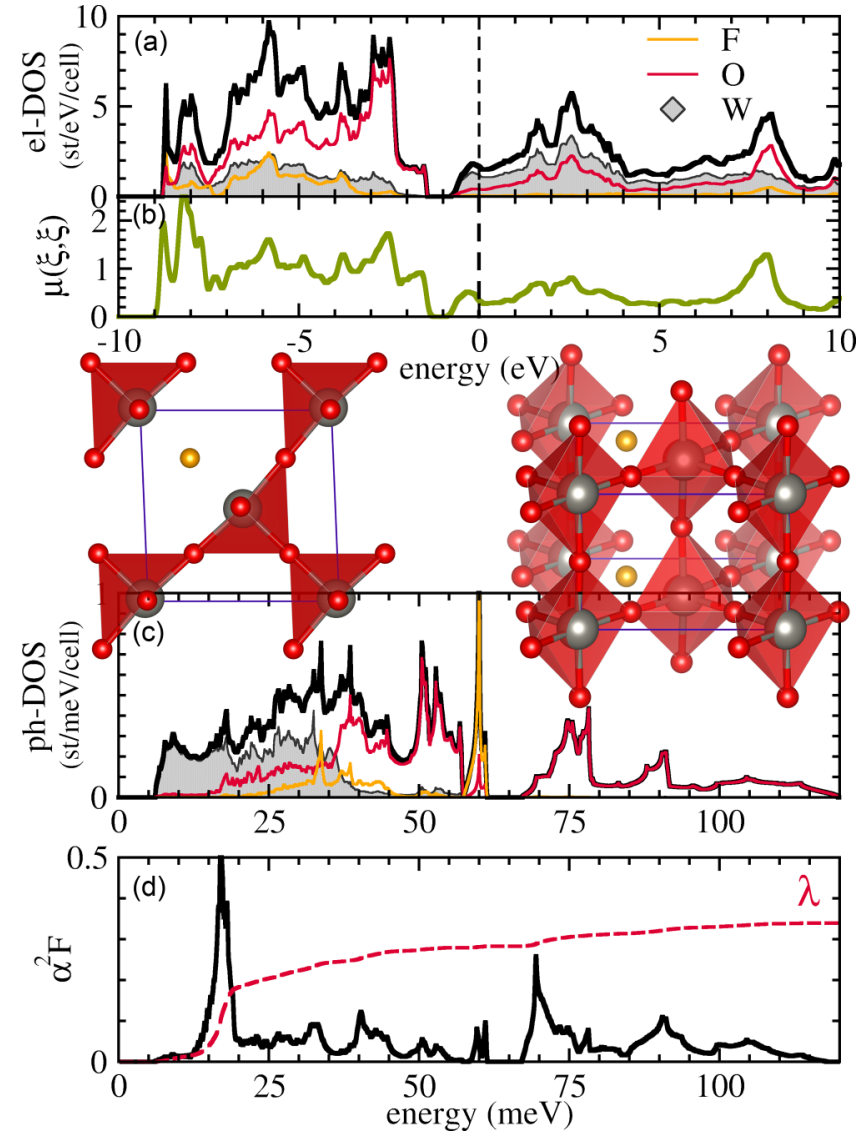

FIG. 3. Crystal structure and collected results for $\mathrm{WO}_{2.5} F_{0.5}$. (a) Electronic density of states (DOS), total (black) and projections onto atomic orbitals [O (red), F (orange), and W (gray)]. (b) Diagonal part of the screened Coulomb interaction $\mu(\xi, \xi)$. (c) Phonon DOS and projections onto atomic species. (d) Eliashberg spectral function $\alpha^{2} \mathrm{~F}$ and electron-phonon interaction parameter $\lambda$. Center: top and side view of the crystal's periodic unit cell.

the occurrence of a sharp low-frequency peak in the $\alpha^{2} \mathrm{~F}$ function due to the soft motion (at finite q) of the apical oxygens [Fig. 3(d)]. The contribution of this peak is crucial for the system to condense into the superconducting state, as it provides alone about one-half of the entire coupling $\lambda$.

The critical temperature, as predicted within SCDFT by treating electron-phonon and Coulomb interactions fully $a b$ initio, is of $0.36 \mathrm{~K}$. The obtained result is in surprisingly good agreement with the experimental value of $0.4 \mathrm{~K}$ measured [10] at similar doping level, $x=0.45$. This comparison, however, should be taken cum grano salis, since the error bar for our SCDFT result is estimated to be quite large for such low values of $T_{c}$, i.e., around $50 \%$ of the value, even for perfectly crystalline systems. We can nevertheless speculate that such a good agreement indicates that the uncertainty in the fluorine distribution and disorder effects are not relevant in the perovskite geometry.

$$
\text { 2. } \mathrm{Cs}_{x} \mathrm{WO}_{3} \text { at } \mathrm{x}=\frac{1}{6}, \frac{1}{3}
$$

Tungsten bronzes are nonstoichiometric compounds with formula $\mathrm{M}_{x} \mathrm{WO}_{3}(0<x<1)$, where $M$ is a metal, usually an alkali. The symmetry of the crystal structure decreases with 

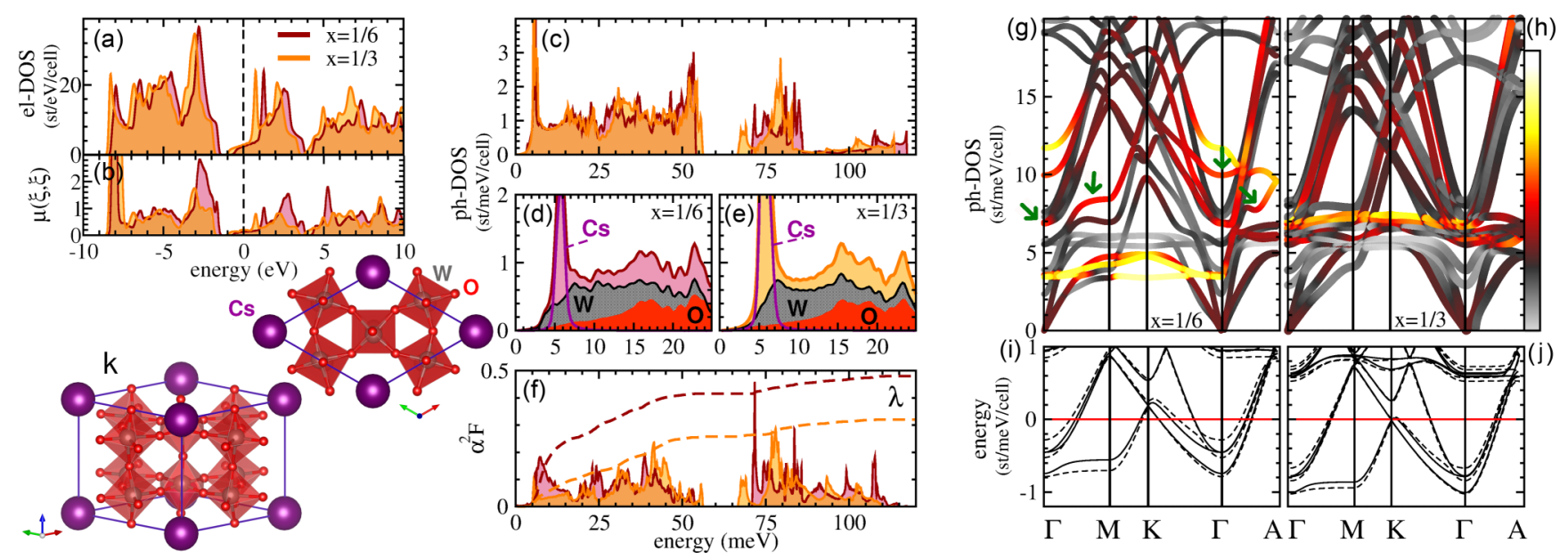

FIG. 4. Collected results for $\mathrm{Cs}_{x} \mathrm{WO}_{3}$ at $x=1 / 3$ and $x=1 / 6$. (e) Electronic density of states (DOS). (b) Diagonal part of the screened Coulomb interaction $\mu(\xi, \xi)$. (c) Phonon DOS. Low-energy part of the phonon DOS resolved onto ionic contributions for (d) $x=1 / 6$ and (e) $x=1 / 3$ [Cs (violet), W (gray), and $\mathrm{O}$ (red)]. (f) Eliashberg spectral function $\alpha^{2} \mathrm{~F}$ and electron-phonon interaction parameter $\lambda$. Low-energy part of the phonon band structure for $(\mathrm{g}) x=1 / 6$ and (h) $x=1 / 3$. The color scale indicates the projection of each mode onto $\mathrm{W}$ components (gray to yellow). Green arrows point at those phonon modes with the strongest coupling. (i,j) Deformation of the band structure for an adiabatic atomic displacement along the most coupled phonon mode (leftmost green arrow in $\mathrm{g}$ ). Bottom left corner, (k) two views of the crystal unit cell for $x=1 / 6$. A second Cs atom (corresponding to $x=1 / 3$ stoichiometry) can be accommodated in the channel between periodic replicas of the shown cell.

the concentration $x$ of the dopant $M$, ranging from cubic to tetragonal (I\&II) to hexagonal. In all these structures the $M$ atoms occupy open channels inside a framework of cornersharing $\mathrm{WO}_{6}$ octahedra. Some of these oxides, with tetragonal or hexagonal symmetry, are known to form superconducting ground states. Experimental data show that the transition temperature $T_{c}$ reaches its maximum in proximity of the insulatormetal transition and then decreases [41] with increasing $x$ $[5,8]$. This is in sharp contrast with the expected behavior of $T_{c}$ from Eq. (4), which predicts higher values upon increasing $N_{E_{F}}$ with the doping concentration. This $T_{c}$ "paradox" has been long discussed in the literature. However, its microscopic origin is not clear, even by the most recent and thorough experimental investigation [8]. Over the last 40 years, several hypothesis $[35,42,43]$ have been formulated. Ngai and Silberglitt [44] have proposed a model for $\mathrm{Na}_{x} \mathrm{WO}_{3}$ in which the tunneling between the semiconducting and superconducting tetragonal structures is assisted, with decreasing $x$, by the softening of a phonon mode corresponding to the rotation of the $\mathrm{WO}_{6}$ octahedra. Bevolo et al. [36], based on heat capacity measurements, and Kamitakahara et al. [42], based on neutron-scattering data, have suggested that this effect might be caused by the looser binding of the $M$ atoms that, owing to the weak-coupling nature of the superconducting state, strongly enhance $T_{c}$, in spite of a weak $x$ dependence of the $M$-atom vibrational energy. Completely different is the analysis performed by Ranninger and Sinha [45] that, assuming a constant deformation potential and lattice dynamics, relates the anomalous behavior of $T_{C}$ to a modulation of the Fermi DOS $N_{E_{F}}$. More recently, a theoretical study by Brusetti et al. [43] of $\mathrm{RbWO}_{3}$, combined with magnetic susceptibility and inelastic neutron-scattering measurements, has excluded all the above-mentioned scenarios. Specifically, the authors have pointed out that the enhancement of $T_{c}$ is not due to an increase in $N_{E_{F}}$, nor to phonon softening (the $\mathrm{Rb}$ modes are weakly coupled, and there is no evidence of soft modes). Their conclusion is that the $T_{c}$ anomaly has to be attributed to a change in the lattice dynamics, which should occur above $30 \mathrm{meV}$.

In this paper we present a computational study [46] of the Cs-doped hexagonal tungsten bronze $[6,35]$. The primitive cell of $\mathrm{Cs}_{x} \mathrm{WO}_{3}$ contains six $\mathrm{WO}_{3}$ f.u. and can accommodate one or two Cs atoms, so that we can simulate the doping levels $x=1 / 3$ and $x=1 / 6$ [47]. The obtained crystal structures, density of states, phonon band structures, Coulomb interactions, and electron-phonon couplings for the systems with these Cs contents are shown in Fig. 4.

The gross features of the electronic and phononic DOS upon $\mathrm{Cs}$ doping are similar to the results discussed for the $\mathrm{F}$ and field-doped $\mathrm{WO}_{3}$ systems in the previous sections. As Cs atoms transfer their valence electron to the $\mathrm{WO}_{3}$ matrix, the Fermi level is shifted into the conduction band. The system becomes metallic with good screening properties, as indicated by the low values of the Coulomb function $\mu$ for $\xi=\xi^{\prime} \simeq 0$ [Fig. 4(b)]. At $x=1 / 6$ the computed DOS at the Fermi level is about 2.33 states/(eV spin). It raises by $25 \%$ of the value at $x=1 / 3$. This result is consistent with the analysis in Ref. [43] and rules out the scenario proposed by Ranninger and Sinha [45] of a $T_{c}$ anomaly driven by a splitting of the Fermi DOS into two maxima. The states at the Fermi level are not hybridized with $\mathrm{Cs}$ states: they are localized on the $\mathrm{WO}_{3}$ tetrahedra and have similar atomic projections $(2 / 3$ on $\mathrm{W}$ and $1 / 3$ on $\mathrm{O}$ ) as in Fig. 3(a). As a consequence, Cs phonon modes do not scatter electrons at the Fermi level and do not provide any relevant superconducting coupling. These modes form an Einstein peak at about $5.5 \mathrm{meV}$, which is clearly visible in Figs. 4(c)-4(e). However, this peak does not give rise to a corresponding structure in the $\alpha^{2} F$ function in Fig. 4(f). It 
can thus be argued, again in agreement with Ref. [43], that Cs ions do not play an active role in the superconducting condensation of the system, which negates the explanation separately proposed by Bevolo et al. and Kamitakahara et al. $[36,42]$.

Nevertheless, the calculated critical temperature shows an excellent quantitative agreement with the experimental trend as a function of the Cs content (see Fig. 2). The qualitative explanation of this effect involves two factors. The leading contribution to the electron-phonon coupling $\lambda$ comes from $\mathrm{W}-\mathrm{O}$ modes, which are mostly associated with zigzagging vibrations of the oxygen octahedra along the $\mathrm{O}_{a p}$ chain. These vibration modes soften due to the opening of the channels and are strongly affected by the doping level. At low Cs content the modes are dispersive in the $7-12 \mathrm{meV}$ range [see Fig. 4(g), green arrows point at the modes with stronger electron-phonon coupling], whereas at maximal doping $x=\frac{1}{3}$ they merge into a structure around $7 \mathrm{meV}$, which also contains some rattling $\mathrm{W}$ modes. More importantly, as the doping level increases, these W-O modes are screened by the extra available charge so that the corresponding contribution to the electron-phonon coupling drops. This last statement follows directly from deformation potential theory [48,49], which links the electron-phonon coupling to the band-structure splitting caused by an adiabatic displacement along the phonon mode [see Figs. 4(i) and 4(j)]. Here, the displacement corresponds to the mode at $q=\Gamma$ marked by a green arrow in Fig. 4(g). Specifically, the resulting electron-phonon matrix element $g_{v}$ at $x=1 / 6$ is more than $30 \%$ larger than that $x=1 / 3$, with a huge effect on $\alpha^{2} \mathrm{~F}$ [Fig. 4(f)] and clearly on $T_{c}$.

It is worth noting that this composition-dependent phonon softening differs from that assumed in Ref. [44] in two aspects: first because the engaged modes do not soften at the transition to the superconducting phase, but, on the contrary, their energy is lower at higher $x$ (although relatively little, so that this effect does not compensate for the much weaker electron-phonon coupling strength); second because the modes are not related to the dynamics of the phase transition, although, like any other structural property of the system, they are affected by it. We emphasize that the key aspect for the observed trend in $T_{c}$ is that the structure of tungsten bronzes is characterized by channels that shift the $\mathrm{WO}_{3}$ octahedral modes to lower energy as compared to the perovskite structure, significantly increasing their coupling. This does not occur in the perovskite $\mathrm{WO}_{3} \mathrm{~F}_{0.5}$, where the oxygen contribution to the phonon DOS starts above $15 \mathrm{meV}$ [see Fig. 3(c)], and $T_{c}$ is 1 order of magnitude lower.
On the other hand, our result also contradicts Ref. [43] on the final statement that the $T_{c}$ anomaly should be caused by high-energy phonons above $30 \mathrm{meV}$. In fact, that conclusion has been drawn from the evidence that no signature of anomaly could be seen in the phonon spectrum computed or measured below that energy. In this regard, we must comment that the phonon softening that we observe is very subtle. Since only few (strongly coupled) modes are involved, the effect is barely visible in the phonon DOS of Figs. 4(c)-4(e). On the other hand, this is evident in the vibrational band structure and the electron-phonon coupling function [Fig. 4(f)], where at $x=\frac{1}{6}$ about one-third of the coupling originates from a small energy window of less than $10 \mathrm{meV}$.

\section{SUMMARY AND CONCLUSION}

By means of parameter-free $a b$ initio SCDFT we have characterized the superconducting state of a set of doped tungsten oxides. The simulated critical temperatures are in excellent agreement with the experimental data, indicating that our theoretical description captures all key aspects of superconductivity in these systems.

It was seen that doped tungsten oxides have to be understood as weak-coupling superconductors, where doping provides carriers to form Cooper pairs but also alters the lattice dynamics, leading to a larger coupling strength in the bronze geometry. Since the coupling is weak, the relevant modes are the lowest energy modes with a significant deformation potential; these can be identified with optical vibrations involving the entire $\mathrm{W}-\mathrm{O}$ octahedra.

The highest critical temperatures are reached in the tungsten bronze structure. Here, $T_{c}$ is enhanced by the opening of channels (containing the dopant) that lower the vibrational energy of the W-O octahedra. The coupling of these modes is larger at low doping: as additional charge is introduced into the system, the modes are screened and their coupling strength decreases. This mechanism explains at a microscopic level the long-debated $T_{c}$ anomaly in tungsten bronzes, where paradoxically, $T_{c}$ is higher at low doping, in spite of a lower carrier density.

In our analysis we have not spotted any signature of strong electron-phonon coupling that could lead, for some doping levels or small structural changes, to $T_{c} \mathrm{~s}$ significantly higher than those measured in tungsten bronzes $(\ll 10 \mathrm{~K})$. If superconductivity on $\mathrm{WO}_{3}$ surfaces and interfaces is confirmed, from our investigation, we expect its origin to be completely different from that in bulk and possibly unconventional (nonphononic).
[1] S. K. Deb, Sol. Energy Mater. Sol. Cells 92, 245 (2008), Selected Papers from the Seventh International Meeting on Electrochromism (IME-7).

[2] J. Su, L. Guo, N. Bao, and C. A. Grimes, Nano Lett. 11, 1928 (2011).

[3] C. ViolBarbosa, J. Karel, J. Kiss, O.-d. Gordan, S. G. Altendorf, Y. Utsumi, M. G. Samant, Y.-H. Wu, K.-D. Tsuei, C. Felser, and S. S. P. Parkin, Proc. Natl. Acad. Sci. U.S.A. 113, 11148 (2016).
[4] C. J. Raub, A. R. Sweedler, M. A. Jensen, S. Broadston, and B. T. Matthias, Phys. Rev. Lett. 13, 746 (1964).

[5] F. Hubble, J. M. Gulick, and W. Moulton, J. Phys. Chem. Solids 32, 2345 (1971).

[6] A. Sweedler, C. Raub, and B. Matthias, Phys. Lett. 15, 108 (1965).

[7] H. R. Shanks, Solid State Commun. 15, 753 (1974).

[8] N. Haldolaarachchige, Q. Gibson, J. Krizan, and R. J. Cava, Phys. Rev. B 89, 104520 (2014). 
[9] J. D. Bocarsly, D. Hirai, M. N. Ali, and R. J. Cava, Europhys. Lett. 103, 17001 (2013).

[10] D. Hirai, E. Climent-Pascual, and R. J. Cava, Phys. Rev. B 84, 174519 (2011).

[11] S. Reich and Y. Tsabba, Eur. Phys. J. B 9, 1 (1999).

[12] S. Reich, G. Leitus, R. Popovitz-Biro, A. Goldbourt, and S. Vega, J. Supercond. Novel Magn. 22, 343 (2009).

[13] A. Palnichenko, O. Vyaselev, A. Mazilkin, I. Zver'kova, and S. Khasanov, Physica C (Amsterdam) 534, 61 (2017).

[14] G. A. de Wijs, P. K. de Boer, R. A. de Groot, and G. Kresse, Phys. Rev. B 59, 2684 (1999).

[15] H. Hamdi, E. K. H. Salje, P. Ghosez, and E. Bousquet, Phys. Rev. B 94, 245124 (2016).

[16] P. Giannozzi, S. Baroni, N. Bonini, M. Calandra, R. Car, C. Cavazzoni, D. Ceresoli, G. L. Chiarotti, M. Cococcioni, I. Dabo, A. D. Corso, S. de Gironcoli, S. Fabris, G. Fratesi, R. Gebauer, U. Gerstmann, C. Gougoussis, A. Kokalj, M. Lazzeri, L. Martin-Samos et al., J. Phys.: Condens. Matter 21, 395502 (2009).

[17] L. N. Oliveira, E. K. U. Gross, and W. Kohn, Phys. Rev. Lett. 60, 2430 (1988).

[18] M. Lüders, M. A. L. Marques, N. N. Lathiotakis, A. Floris, G. Profeta, L. Fast, A. Continenza, S. Massidda, and E. K. U. Gross, Phys. Rev. B 72, 024545 (2005).

[19] M. A. L. Marques, M. Lüders, N. N. Lathiotakis, G. Profeta, A. Floris, L. Fast, A. Continenza, E. K. U. Gross, and S. Massidda, Phys. Rev. B 72, 024546 (2005).

[20] A. Linscheid, A. Sanna, A. Floris, and E. K. U. Gross, Phys. Rev. Lett. 115, 097002 (2015).

[21] J. A. Flores-Livas and A. Sanna, Phys. Rev. B 91, 054508 (2015).

[22] A. Sanna, in The Physics of Correlated Insulators, Metals, and Superconductors, edited by E. Pavarini, E. Koch, R. Scalettar, and R. Martin (Verlag des Forschungszentrum Jülich, Jülich, 2017), Vol. 7, Chap. 16, p. 429.

[23] S. Kurth, Ph.D. thesis, Bayerische Julius-Maximilians Universität Würzburg, 1995.

[24] The ELK FP-LAPW code, http://elk.sourceforge.net/.

[25] A. Sanna, J. A. Flores-Livas, A. Davydov, G. Profeta, K. Dewhurst, S. Sharma, and E. K. U. Gross, J. Phys. Soc. Jpn. 87, 041012 (2018).

[26] P. Morel and P. W. Anderson, Phys. Rev. 125, 1263 (1962).

[27] D. J. Scalapino, J. R. Schrieffer, and J. W. Wilkins, Phys. Rev. 148, 263 (1966).

[28] P. B. Allen and B. Mitrović, Theory of Superconducting Tc, Solid State Physics Vol. 37 (Academic Press, New York, 1983), pp. 1-92.
[29] W. L. McMillan, Phys. Rev. 167, 331 (1968).

[30] P. B. Allen and R. C. Dynes, Phys. Rev. B 12, 905 (1975).

[31] K. Ueno, S. Nakamura, H. Shimotani, H. T. Yuan, N. Kimura, T. Nojima, H. Aoki, Y. Iwasa, and M. Kawasaki, Nat. Nanotechnol. 6, 408 (2011).

[32] J. Jeong, N. Aetukuri, T. Graf, T. D. Schladt, M. G. Samant, and S. S. P. Parkin, Science 339, 1402 (2013).

[33] A. D. Walkingshaw, N. A. Spaldin, and E. Artacho, Phys. Rev. B 70, 165110 (2004).

[34] In the tetragonal electron-doped systems (8-atom unit cells) we integrate the Brillouin zone with an $8^{3} \mathbf{k}$ grid and $4^{3} \mathbf{q}$ grid for electrons and phonons, respectively. The grids are reduced to $6^{3}$ and $2^{3}$ for the larger (monoclinic) hole-doped unit cell.

[35] M. R. Skokan, W. G. Moulton, and R. C. Morris, Phys. Rev. B 20, 3670 (1979).

[36] A. J. Bevolo, H. R. Shanks, P. H. Sidles, and G. C. Danielson, Phys. Rev. B 9, 3220 (1974).

[37] J. Zhu, M. Vasilopoulou, D. Davazoglou, S. Kennou, A. Chroneos, and U. Schwingenschlögl, Sci. Rep. 7, 40882 (2017).

[38] X. Leng, J. Pereiro, J. Strle, G. Dubuis, A. T. Bollinger, A. Gozar, J. Wu, N. Litombe, C. Panagopoulos, D. Pavuna, and I. Bozovic, npj Quantum Materials 2, 35 (2017).

[39] J. Meyer, S. Hamwi, S. Schmale, T. Winkler, H.-H. Johannes, T. Riedl, and W. Kowalsky, J. Mater. Chem. 19, 702 (2009).

[40] Experimentally, fluorine doping leads to a (disordered) cubic symmetry; therefore the theoretical inequivalence of the two sites is irrelevant.

[41] Some of the hexagonal tungsten bronzes (HTB) show a reentrant semiconducting behavior interrupting the trend.

[42] W. A. Kamitakahara, K. Scharnberg, and H. R. Shanks, Phys. Rev. Lett. 43, 1607 (1979).

[43] R. Brusetti, P. Bordet, J. Bossy, H. Schober, and S. Eibl, Phys. Rev. B 76, 174511 (2007).

[44] K. L. Ngai and R. Silberglitt, Phys. Rev. B 13, 1032 (1976).

[45] J. Ranninger and K. P. Sinha, Proc. Indian Acad. Sci., Chem. Sci. 95, 93 (1985).

[46] We integrate the Brillouin zone with an $8^{3} \mathbf{k}$ grid and $4^{3} \mathbf{q}$ grid for electrons and phonons, respectively.

[47] Note that in the latter case disorder effects like the Anderson localization are not taken into account. This phenomenon was invoked to explain a reentrant insulating state in $\mathrm{Ba}_{x} \mathrm{WO}_{3}$, which does not occur in the Cs compound.

[48] F. S. Khan and P. B. Allen, Phys. Rev. B 29, 3341 (1984).

[49] W. Jones and N. H. March, Theoretical Solid State Physics (Dover Publications, New York, 1973). 